\title{
Evaluating cochlear insertion trauma and hearing preservation after cochlear implantation (CIPRES): A study protocol for a randomised single blind controlled trial
}

\section{Saad Jwair ( $\nabla$ s.jwair@umcutrecht.nl )}

Universitair Medisch Centrum Utrecht https://orcid.org/0000-0001-8027-0681

\section{Ralf Boerboom}

Universitair Medisch Centrum Utrecht

Huib Versnel

Universitair Medisch Centrum Utrecht

Robert Stokroos

Universitair Medisch Centrum Utrecht

Hans Thomeer

Universitair Medisch Centrum Utrecht

\section{Study protocol}

Keywords: Cochlear implant, Insertion trauma, Electrode array, Lateral wall, Perimodiolar, Round window, Cochleostomy, Scalar translocation, Electrocochleography

Posted Date: November 5th, 2020

DOI: https://doi.org/10.21203/rs.3.rs-36735/v1

License: (c) (i) This work is licensed under a Creative Commons Attribution 4.0 International License. Read Full License

Version of Record: A version of this preprint was published at Trials on December 1st, 2021. See the published version at https://doi.org/10.1186/s13063-021-05878-2. 


\section{Abstract}

\section{Background}

In order to preserve residual hearing in patients with sensorineural hearing loss (SNHL) who receive a cochlear implant $(\mathrm{Cl})$, insertion trauma to the delicate structures of the cochlea needs to be minimized. The surgical approach comprises the conventional mastoidectomy-posterior tympanotomy (MPT) to arrive at the middle ear, followed by either a cochleostomy (CO) or the round window (RW) approach. Both techniques have their benefits and disadvantages. Another important aspect in structure preservation is the design of the electrode array. Two different designs are used: a 'straight' lateral wall lying electrode array (LW), or a 'pre-curved' perimodiolar lying electrode array (PM). Interestingly, until now, the best surgical approach and design of the implant is uncertain. Our hypothesis is that the combination of a RW approach and a LW lying electrode array minimizes insertion trauma, leading to better hearing outcome for SNHL patients.

\section{Methods}

We designed a monocenter, $2 \times 2$, randomized controlled trial to compare insertion trauma between four groups of patients, with each group having a unique combination of electrode array type (LW or PM) and surgical approach (RW or $\mathrm{CO}$ ). In total, 48 patients will be randomized into one of these four intervention groups. Our primary objective is comparison of postoperative hearing preservation between these four groups. Secondly, we aim to assess structure preservation (i.e., scalar transposition, with basilar membrane disruption or tip fold-over of array) for each group. Thirdly, we will compare objective outcomes of hearing and structure preservation by way of electrocochleography (ECochG).

\section{Discussion}

Cochlear implantation by way of a cochleostomy or round window approach, using different electrode array types, is the standard medical care for patients with severe to profound bilateral sensorineural hearing loss, as it is a relative simple and low-risk procedure that greatly benefits patients. However, loss of residual hearing remains a problem. This trial is the first randomized controlled trial that evaluates the effect of cochlear insertion trauma of several $\mathrm{Cl}$ treatment options on hearing preservation.

\section{Trial registration}

This trial is registered in the Netherlands Trial Register (NTR) (www.trialregister.nl): NL8586, date of registration: $4^{\text {th }}$ of May 2020. Retrospectively registered; $3 / 48$ participants were included before registration.

\section{Introduction}

\section{Background and rationale $\{6 a\}$}


In people with severe sensorineural hearing loss or deafness, hearing can be (partially) restored with a cochlear implant $(\mathrm{Cl})$. A Cl bypasses the sensory hair cells and directly stimulates the auditory nerve via electrical current pulses, allowing deaf patients to hear again. Cochlear implantation has become a standard and accepted treatment for severely hearing impaired patients throughout the years in high income countries. Hearing with a $\mathrm{Cl}$ has seen a tremendous development in auditory perception, from only sound detection in the 1980s to speech understanding in the last decades (1). However, speech understanding is far from optimal, especially in difficult situations where background noise is present, and perception of other sounds as music can also be quite troublesome. Several studies have shown that preserving residual hearing can lead to better hearing outcomes, especially in noisy environments (2-5). In order to preserve residual hearing, trauma to the delicate structures of the cochlea needs to be minimized during the surgical implantation procedure.

The surgical procedure commonly starts with the conventional mastoidectomy-posterior tympanotomy (MPT) approach to the middle ear, and is followed by accessing the cochlea, either through a cochleostomy ( $\mathrm{CO}$ ) or the round window (RW). Several papers, including systematic reviews comparing $\mathrm{CO}$ and RW approaches in literature, concluded that evidence lacks regarding preference for one or the other approach with respect to hearing preservation (6-10). Both techniques of accessing the cochlea have their potential pros and cons (e.g., cochleostomy leads to a smaller angle of insertion and by definition induces damage to outer bony wall and spiral ligament, while the RW approach ensures a correct positioning of the electrode array and leaving outer bony wall and spiral ligament intact). The extended round window (ERW) approach will not be tested in this study. The ERW approach is a combination of the direct RW and cochleostomy approach, and is generally considered to be a variant of the cochleostomy approach. It is therefore unlikely that the ERW approach would be significantly different than the cochleostomy approach. One may also argue a preference for a certain approach based on individual cochlear structures. Several studies for example have clearly shown that each human cochlea has a different 'cochlear hook' in parallel with one's unique fingerprint (11-13).

Correct insertion, for both cochleostomy and round window approach, ensures that the implant is in the scala tympani of the cochlea (14). If during insertion, the $\mathrm{Cl}$ translocates to the scala vestibuli or scala media, the basilar membrane with the organ of Corti (the physiological receptor organ that transduces the acoustic energy) is damaged. Scalar translocation can negatively influence the final hearing outcome and hearing preservation of $\mathrm{Cl}$ patients $(15,16)$.

Another aspect relevant for minimizing insertion trauma, is the design of the electrode array. There are two fundamentally different designs: a 'straight' lateral wall lying electrode array (LW), or a 'pre-curved' perimodiolar lying electrode array (PM). No evidence has been provided that one design outperforms the other in terms of hearing with a $\mathrm{Cl}$ and structure preservation $(7,10,15)$. On the one hand, lateral wall positioning might be the best way to preserve structures as the osseous spiral lamina, and basilar membrane; on the other hand, perimodiolar positioning might provide better hearing with a $\mathrm{Cl}$ (which is the ultimate objective for deaf patients with a $\mathrm{Cl}$ ), as the electrodes are situated closer to the medially situated spiral ganglion cells which form the auditory nerve and need to be electrically stimulated. In 
addition, the perimodiolar array has the potential to minimize contact between the array and the lateral wall, leading to structure preservation of the lateral wall and stria vascularis.

According to one study, speech perception scores were better for the LW group (17). On the contrary, other studies report better speech perception outcomes for the PM group $(7,15)$. The majority of the studies, however, showed no difference between both groups (18-22). However, all these studies had a high risk of bias. In addition, the studies failed to differentiate between the surgical approaches, inducing a major confounding factor.

It is unclear which surgical approach and electrode design is most suited to achieve minimal insertion trauma, and thereby preserving residual hearing in cochlear implantation surgery. Therefore, it is not surprising that worldwide both type of approaches and electrode designs are used.

Considering the surgical approach and electrode array design, it is important to note that during insertion no reliable feedback is provided regarding the array tip position in relation to the intracochlear structures. After inserting the tip of the electrode array in the round window perforation or cochleostomy, only tactile feedback is available which might not be sufficient to distinguish whether the implant is correctly inserted.

One of the possibilities to view the intracochlear structures and thereby discern the scalar location of the array in relation to the micro-anatomical structures (thus providing postoperative feedback), is by applying imaging techniques after surgery such as cone beam computed tomography (CB-CT), which has been proven to be reliable in differentiating the different scalae and exact electrode array position (23-25). Another possibility to detect insertion trauma is by intraoperative electrophysiological measurements, providing indirect feedback: intracochlear electrocochleography (ECochG) which measures responses of residual functioning hair cells and spiral ganglion cells to acoustic tone stimuli. During insertion, ECochG measures can be used to assess the probability of insertion trauma, thus providing feedback of the insertion (26-29).

\section{Objectives $\{7\}$}

The primary objective of this study is to compare hearing preservation after cochlear implantation between the four possible combinations of surgical approaches ( $\mathrm{CO}$ and $\mathrm{RW}$ ) and electrode array designs (LW and PM). Hearing preservation will be measured postoperatively with pure tone audiometry. Secondary objectives are to compare the effect of these interventions on scalar position and ECochG measures. Furthermore, we aim to assess the relationship between the outcome measures for hearing preservation (audiometry, ECochG and postoperative $\mathrm{CT}$ ).

\section{Trial design $\{8\}$}

This study concerns a single-blind mono-center 2x2 factorial designed RCT. All four treatment options are implemented interchangeably in standard medical care, however our hypothesis is that the combination of RW and LW is superior to the other interventions. Participants will be blinded for surgical 
approach/type of electrode. In total, 48 participants will be included, all groups carry the same equal weight (allocation ratio 1:1:1:1).

\section{Methods: Participants, Interventions And Outcomes}

\section{Study setting $\{9\}$}

This is a mono center study performed at the department of Otorhinolaryngology in the University Medical Center Utrecht, an academic hospital, and is expected to run for approximately three years.

\section{Eligibility criteria $\{10\}$}

All participants will undergo the usual standard medical care of work-up before, during and after during cochlear implantation. The work-up includes a pure tone audiogram (PTA), a speech audiogram, a preoperative $\mathrm{CT}$, and interviews with speech therapist, audiologist, ENT surgeon and social worker. In a multidisciplinary meeting, the Cochlear implantation team of the UMC Utrecht will assess all results and decide whether a patient is eligible for a $\mathrm{Cl}$. In addition, according to standard medical care, participants will receive corticosteroids before and after surgery.

\section{Inclusion Criteria}

- Dutch language proficiency

- 18 years or older

- Choice for Advanced Bionics implant

- No signs of acute or chronic middle ear infections and/or mastoiditis

\section{Exclusion Criteria}

- Prior otologic surgery in the implanted ear (excluding tympanostomy tube placement)

- Inner ear malformation (i.e. ossification, Mondini malformation)

- Retrocochlear pathology

- Neurocognitive disorders

- Acute or Chronic otomastoiditis

\section{Who will take informed consent? $\{26 a\}$}

Participants ENT-physician or audiologist during visits to the outpatient clinic will asks whether the patient would be interested to participate in the study. Additional verbal and written information about the study will be provided to all participants by an investigator. An investigator will also provide and obtain 
the informed consent (IC) form, which is also co-signed by the investigator. There will be ample opportunity (at least 1 week) for the participants to consider participation and discuss their questions with one of the investigators before the participants may decide to sign the IC form in order to participate. Participation in the study is entirely voluntary. If a subject wants to participate, several appointments for the audiological follow-up will be scheduled. If a patient does not want to participate, contact with the investigator will be terminated.

\section{Additional consent provisions for collection and use of participant data and biological specimens $\{26 \mathrm{~b}\}$}

Not applicable, no additional consent is required for use of participant data and biological specimens.

\section{Interventions}

\section{Explanation for the choice of comparators $\{6 \mathrm{~b}\}$}

Four groups of participants will be included, which all have a different combination of electrode type, and surgical insertion approach. These treatment options are all standard care in cochlear implants centers worldwide.

\section{Intervention description $\{11 \mathrm{a}\}$}

The electrode type consists of either a lateral wall electrode array, or a perimodiolar electrode array, specifically, respectively the SlimJ and Midscala electrode arrays. Both these arrays are developed by Advanced Bionics. Two surgical approaches are used, a round window or cochleostomy approach. The cochleostomy is placed antero-inferiorly from the round window niche.

\section{Criteria for discontinuing or modifying allocated interventions $\{11 \mathrm{~b}\}$}

It is only possible to change the allocated intervention via a second surgery, by removing the cochlear implant. A second surgery increases potential harm for the patient, outweighing potential benefits. Therefore, removal of the cochlear implant is only performed if medically necessitated, e.g. in instances of malfunctioning device, wound infection or persisting pain. Such rare cases will be discussed in a plenary session dedicated for cochlear implant patients, in line with normal standard medical care.

\section{Strategies to improve adherence to interventions $\{11 \mathrm{c}\}$}

To improve adherence to the study protocol, the follow-up measurements for the study are planned simultaneously with the standard medical rehabilitation appointments. Apart from showing up for the follow-up appointments, participants do not need to adhere to specific tasks.

\section{Relevant concomitant care permitted or prohibited during the trial $\{11 \mathrm{~d}\}$}

Not applicable, there is no relevant concomitant care that is permitted or prohibited.

\section{Provisions for post-trial care $\{30\}$}


The sponsor has an insurance which is in accordance with the legal requirements in the Netherlands (Article $7 \mathrm{WMO}$ ). This insurance provides cover for damage to research participants through injury or death caused by the study. The insurance applies to the damage that becomes apparent during the study or within 4 years after the end of the study.

\section{Outcomes $\{12\}$}

At intake baseline data will be collected, including gender, age, duration of deafness, pre- or post-lingually deafened, cause of deafness and side of implantation. In addition, the most recent pure tone thresholds ( $250 \mathrm{~Hz}, 500 \mathrm{~Hz}$ and 1, 2, 4, and $8 \mathrm{kHz}$ ) and speech reception thresholds (SRT) for consonant-vowelconsonant (CVC) word lists in quiet for both ears will be collected.

\section{Hearing preservation}

Hearing preservation is calculated by comparing pure-tone thresholds after and before $\mathrm{Cl}$ surgery using the following equation (30), Eqn. 1:

$$
H P=1-\frac{(\text { PTA post }- \text { PTA pre })}{(\text { PTA max }- \text { PTA pre })}
$$

In this equation, HP is hearing preservation, is the average pure-tone (unaided) hearing threshold of 125 , 250 and $500 \mathrm{~Hz}$ measured preoperatively,PTApost is the same average pure-tone hearing threshold measured postoperatively, and PTAmax is the maximum sound intensity generated by a standard audiometer (usually between 90 and $120 \mathrm{~dB} \mathrm{HL}$ ). With full preservation of hearing, $\mathrm{HP}=100$, and with complete loss of hearing HP=0. The postoperative tone audiometry will also be measured at 3,6 and 12 months after activation of the cochlear implant.

\section{Scalar positioning of the electrode array}

We will use the CB-CT scanner (Newtom VGi EVO, Cefla Italy) to postoperatively assess the scalar location of the electrode array in cochlea's of all four groups. The CB-CT has been proven to be the best imaging modality to date, for assessing the scalar location postoperatively, as it has low radiation artefacts (caused by the metal parts of the cochlear implant) and high spatial resolution needed to image the cochlea and its internal parts. Other advantages of this modality are amongst others that it has relatively low radiation exposure, is less likely to trigger claustrophobic events and requires shorter scanning durations compared to traditional CT scanners (31-33). CB-CT imaging postoperatively leads to exposure of low-dose radiation (effective dose: $0.18 \mathrm{mSv}$ ), and is therefore considered to be of low-risk.

We will assess $\mathrm{Cl}$ translocation by making multiplanar midmodiolar reconstructions of the CB-CT images, which is validated (23-25). These multiplanar reconstructions will allow us to systematically indicate for every electrode contact of the electrode array the exact scalar position (i.e. scala tympani or scala vestibuli). 
Electrocochleography (ECochG) is a method for recording the electrical potentials of the cochlea. The ECoch $G$ is composed of several components: the compound action potential (CAP), auditory nerve neurophonic (ANN), cochlear microphonics (CM) and the summating potential (SP). In essence, the CAP and ANN reflect auditory nerve activity, the $\mathrm{CM}$ and $\mathrm{SP}$ are generated by the hair cells of the organ of Corti. The CM is an alternating current response following the tone, and the SP is a direct current response. Outcome measures include the total ECochG amplitude. Potentially, the difference in the amplitude of the total ECochG response after and before insertion might contain information about insertion trauma, i.e. damage to the basilar membrane, stria vascularis or other structures.

Intraoperatively we will use the most apical contact point of the electrode array to measure these outcomes during insertion. The acoustic pure tones stimuli will be delivered via an earphone (earplug) on the operated ear. This will be coupled to the measurement equipment (active insertion monitoring system, Advanced Bionics) that is provided by the manufacturer. The amplifier in the implant will be used for amplification of the response. Apart from prolonged surgery time (estimate of 10 mins), there will be no added risk for the participant. Postoperatively ECochG measurements will be repeated. We will perform recordings at each of the 16 electrodes for the following frequencies: $125,250,500 \mathrm{~Hz}$ and 1, 2, 3, $4 \mathrm{kHz}$. In addition, acoustic tone thresholds will be indirectly estimated by measures of the total ECoch $\mathrm{G}$ responses.

\section{Speech perception}

One year after activation of the $\mathrm{Cl}$, a conventional speech perception test in quiet and in noise will be performed with CVC words from the 'Nederlandse Vereniging voor Audiologie' (NVA: Dutch Society of Audiology) word-list. Speech reception thresholds will be registered. Also, the clinical spectral ripple test, which uses ripples instead of words, can be used to complement speech perception scores in noise (34).

The extra ECochG measurements and tone/speech tests are not considered to be of any risk for the participants.

\section{Participant timeline $\{13\}$}

Schedule of enrolment, interventions, and assessments is depicted in Figure 1. Participants will be screened and enrolled two weeks before the surgery. On the day of surgery the participant will be allocated to one of the four groups (A-D). During surgery intraoperative ECochG measure will be conducted. The CB-CT scan will be performed on the same day after surgery. Activation of the $\mathrm{Cl}$ is approximately 4-6 weeks after surgery. After activation of the $\mathrm{Cl}$, the first audiometry and postoperative ECochG measures will be conducted. These measurements will be repeated at approximately 2,5 and 12 months after activation of the $\mathrm{Cl}$.

\section{Sample size $\{14\}$}


Hearing preservation as computed according to Eqn. 1 is the main outcome variable. Based on three studies in literature (35-37) we expect a large range of hearing preservation for each group, from 0 (no preservation, i.e., loss of all hearing) to 100 (full preservation, hearing stable), and occasionally above (improved hearing). These three studies showed means of 56,74, and 53, respectively and within-group standard deviations, $\sigma_{W}$, of 37,19 and 37 . Weighing the larger studies $(35,37)$ more we estimate $\sigma_{W}=35$, group means of 50 with clinically interesting differences of 40 between best and worst group means. This yields a variance between groups of 400 , i.e., $\sigma_{B}=\sqrt{ } 400=20\left(\sigma_{B}\right.$ : standard deviation between groups). Thus, effect size $\sigma_{\mathrm{B}} / \sigma_{\mathrm{W}}=0.57$. Using a group size of 12 yields a robust power of 0.9 . We used G*power (version 3.1.9) to calculate the power based on effect size and sample size with a one-way ANOVA test, with fixed effects.

\section{Recruitment $\{15\}$}

No particular strategies were developed to increase the likelihood of participant enrolment. However, in developing the protocol, efforts were made to limit the extra burden for participants participating in this study. For example, follow-up measurements are planned on the same days of rehabilitation appointments. Also, based on previous experience and data, we expect to achieve adequate participant enrolment to reach our targeted sample size.

\section{Assignment of interventions: allocation}

\section{Sequence generation $\{16 \mathrm{a}\}$}

A separate independent department, the Julius Research and Epidemiology Department of the University Medical Center Utrecht, will handle the method of generating the allocation sequence. They will stratify the groups for age, with two subgroups, 18-50 years and equal or more than 50 years. Beforehand, 8 numbers are created, indicating a combination of group (A-D) and stratification (below or equal to 50 and higher than 50 years).

\section{Concealment mechanism $\{16 b\}$}

In a sequentially manner every participant will be allocated randomly a number from 4 numbers that are possible (based on age) by the research tool software of the Julius center and Epidemiology department. The allocation is tied to block randomization. The allocation will be done before surgery, and after IC approval and screening.

\section{Implementation $\{16 c\}$}

The Julius centre and epidemiology department will generate the allocation sequence with their own developed research tool for randomisation. A participant can be included by every member of the research team, when in doubt, the inclusion will be judged by the whole research team. Subsequently, based on the allocation, the patient is assigned to one of the groups by a member of the research team. 


\section{Assignment of interventions: Blinding}

\section{Who will be blinded $\{17 a\}$}

This study is single-blind, meaning that only participants are blinded for the treatment allocation. Because of the nature of the intervention (type of surgery and intracochlearly placed electrode array), it is impossible for the patient to discover the allocation. The research team is not blinded. The audiology assistants, however, who will perform the audiometry, are blinded. In addition, the offline outcome data will be blindly analysed.

\section{Procedure for unblinding if needed $\{17 \mathrm{~b}\}$}

In rare cases in which the device has to be removed via surgery unblinding may be permissible. Before the second surgery, the subject will be unblinded by a member of the research team, who will discuss the treatment options with the subject. If needed, the surgeon will also be informed about the exact intervention, as is standard in medical practice.

\section{Data collection and management}

\section{Plans for assessment and collection of outcomes $\{18 \mathrm{a}\}$}

All data will be collected using an electronic data capture (EDC) tool (Castor EDC). The UMC Utrecht healthcare data of the participants, including baseline outcomes, CT images and results of the audiometry will be derived from the electronic patient file. The assessors are specialized in otorhinolaryngology, and therefore they are trained in assessing the audiology, CT images and electrophysiology data of this study.

\section{Plans to promote participant retention and complete follow-up $\{18 \mathrm{~b}\}$}

Once a participant is enrolled and randomized, the study site will make every reasonable effort to follow the subject for the entire study period. Participants can leave the study at any time for any reason if they wish to do so without any consequences. Participants who withdraw from the study or who terminate the recording sessions prematurely, in the absence of any adverse event, will not be followed. Participant retention will be increased by schedule strategies, e.g. by planning the follow-up measures on the same day of clinical rehabilitation. Participants will also be reminded of the study via e-mail between sessions, including information of any published results (if they are interested).

\section{Data management $\{19\}$}

All data will be handled confidentially and research data will be coded by using a unique patient identification number. To be able to reproduce the study finding and to help future users to understand and reuse the data all changes made to the raw data and all steps taken in the analysis will be documented. The database files will be kept for 15 years after the study has ended. 


\section{Confidentiality $\{27\}$}

The key to the code will be safeguarded by the investigators. All data will be stored on the research network disc of the UMC Utrecht in a secured research folder structure. Only the team of investigators will have access to the database files.

\section{Plans for collection, laboratory evaluation and storage of biological specimens for genetic or molecular analysis in this trial/future use $\{33\}$}

Not applicable; no biological specimens will be collected for this study.

\section{Statistical methods}

\section{Statistical methods for primary and secondary outcomes $\{20 \mathrm{a}\}$}

We will use an ANOVA test to compare the primary outcome measure hearing preservation between the four groups. We will do the same for the SRT thresholds (in quiet and in noise) between the groups. Fisher's exact test of independence will be used to compare the electrode location within the scala tympani (correct location after insertion) between $\mathrm{CO}$ or RW approach, and between PM or LW electrode insertion. We will also use multiple regression analysis to identify independent predictors of hearing preservation. Among the factors to examine are insertion depth and cochlear volume. We will use a Pearson correlation test to examine the correlations between the ECochG responses and hearing preservation at the various time points (during and after cochlear implantation).

\section{Interim analyses $\{21 \mathrm{~b}\}$}

Independent analysis of the Institutional Review Board (IRB) or in Dutch 'Medisch Ethische Toetsing Commissie' (METC) classified this study as a low risk, not needing a data safety monitoring committee (DSMC), mainly because all interventions are standard medical care. Therefore, no interim analyses will be conducted during this trial.

\section{Methods for additional analyses (e.g. subgroup analyses) \{20b\}}

No additional analyses will be conducted. Participants age might play an important role in outcomes, however the randomization procedures stratifies for age for every intervention group (A-D).

\section{Methods in analysis to handle protocol non-adherence and any statistical methods to handle missing data $\{20 \mathrm{c}\}$}

Participants who withdraw from the study or who terminate the recording session prematurely will be considered as lost and will be replaced. Reasons for withdrawal or premature termination will be documented. We expect a withdrawal rate of participants of no more than $10 \%$ (since $N=48$, this is 5 ). The number of replacements will be limited to two persons per subgroup. Depending on the missing values, multiple imputation or simply list wise deletion will be conducted for the missing values. 
Plans to give access to the full protocol, participant level-data and statistical code $\{31 \mathrm{c}\}$

Data sharing, including full protocol, participant datasets and statistical codes will be considered upon reasonable request.

\section{Oversight and monitoring}

\section{Composition of the coordinating centre and trial steering committee $\{5 \mathrm{~d}\}$}

Trial quality will be independently monitored by a local monitor (UMC Utrecht) once a year. The local monitor will check at least $10 \%$ of the signed ICs. From the first five participants the in- and exclusion criteria will also be checked. The monocenter study file will be also monitored. This study has no public involvement group.

\section{Composition of the data monitoring committee, its role and reporting structure $\{21 \mathrm{a}\}$}

Not applicable, a data monitoring committee is not appointed for this study.

\section{Adverse event reporting and harms $\{22\}$}

Adverse events (AE) will be recorded; serious adverse events (SAE) will be reported to the local IRB, and centrally stored in a digital database. Serious adverse events are not expected, but in case they do occur, the research group can decide to terminate prematurely the study.

\section{Frequency and plans for auditing trial conduct $\{23\}$}

The investigators will submit a summary of the progress of the trial to the accredited IRB once a year. Information will be provided on the date of inclusion of the first subject, numbers of participants included and numbers of participants that have completed the trial, serious adverse events/serious adverse reactions, protocol violations, other problems, and amendments.

\section{Plans for communicating important protocol amendments to relevant parties (e.g. trial participants, ethical committees) $\{25\}$}

Amendments are changes made to the research after a favourable opinion by the accredited IRB has been given. All protocol amendments will be notified to the IRB for approval. Non-substantial amendments will not be notified to the accredited IRB and the competent authority, but will be recorded and filed by the sponsor.

\section{Dissemination plans $\{31$ a $\}$}

The trial results will be made accessible to the public in a peer-review journal, preferable in an open access-study journal. In addition, key trial results will be presented in national and international conferences and other relevant meetings. There are no publication restrictions. 


\section{Discussion}

Cochlear implantation by way of a cochleostomy or round window approach, using different electrode array types, is the standard medical care for patients with severe to profound bilateral sensorineural hearing loss, as it is a relative simple and low-risk procedure that greatly benefits patients. Despite the increased interest in hearing preservation, loss of residual hearing remains an important problem in cochlear implantations. This might be caused by a lack of adequate, randomized, and blinded prospective studies, investigating hearing preservation in $\mathrm{Cl}$ patients. There are studies that investigated hearing preservation in $\mathrm{Cl}$ patients, however these studies have a high risk of bias. Therefore, the level of evidence for many aspects of hearing preservation is low. This trial is the first prospective, randomized controlled trial that evaluates the effect of cochlear insertion trauma of several $\mathrm{Cl}$ treatment options on hearing preservation. Another strength of this study, is evaluation of insertion trauma by three separate assessment tools: audiometry, electrophysiology and CT imaging. These tools can complement each other, potentially leading even to detection of minimal insertion trauma. In addition, the multiple outcome measures allow us to investigate insertion trauma on the short and long term.

\section{Trial status}

Protocol Version 3, 02-01-2020. Date of first recruitment: 31-01-2020. Currently 3/48 participants are included, date: 12-06-2020. Approximate date of trial completion: 31-01-2023.

\section{Abbreviations}




\begin{tabular}{ll} 
AE & Adverse Event \\
\hline CB-CT & Cone beam computed tomography \\
\hline CO & Cochleostomy approach \\
Cl & Cochlear Implant \\
\hline CVC & Consonant-Vowel Nucleus-Consonant, dutch speech perception test \\
\hline ECochG & Intracochlear electrocochleography \\
\hline IC & Informed Consent \\
\hline IRB & Institutional Review Board \\
\hline LW & Lateral wall electrode array \\
\hline METC & $\begin{array}{l}\text { Medical research ethics committee (MREC); in Dutch: medisch ethische toetsing } \\
\text { commissie (METC) }\end{array}$ \\
\hline
\end{tabular}

MPT Mastoidectomy-posterior tympanotomy

PTA Pure tone audiometry

PM Perimodiolar electrode array

RW Round window approach

(S)AE (Serious) Adverse Event

SRT Speech Reception Threshold

SNHL Sensorineural hearing loss

WMO Medical Research Involving Human Participants Act; in Dutch: Wet Medischwetenschappelijk Onderzoek met Mensen

\section{Declarations}

\section{Acknowledgements}

Not applicable

\section{Authors' contributions $\{31 \mathrm{~b}\}$}

All named authors adhere to the authorship guidelines of Trials. SJ developed the protocol, drafted the manuscript, revised the manuscript and approved the final version. HT, RB, HV and RS developed the protocol, revised the manuscript and approved the final version. No other writers were involved.

\section{Funding $\{4\}$}


Advanced Bionics funds this study in total by providing the funds for the $\mathrm{PhD}$ project of the main author (SJ) and funding of necessary laboratory material. The funding sources had no role in the design and conduct of the study; collection, management, analysis, and interpretation of the data; preparation, review, or approval of the manuscript; and decision to submit the manuscript for publication.

\section{Availability of data and materials $\{29\}$}

There are no limitations limiting access to the dataset. The research team has complete access to all data of this study.

\section{Ethics approval and consent to participate $\{24\}$}

The study will be conducted according to the principles of the 64th WMA General Assembly in Fortaleza (Brazil, October 2013), and in accordance with the Medical Research Involving Human Participants Act (WMO).

This research protocol was approved by the Institutional Review Board (IRB) of the UMC Utrecht (METC 19-700, version 3, 14-01-2020). Written, informed consent to participate will be obtained from all participants.

\section{Consent for publication $\{32\}$}

See supplementary materials for the IC form.

\section{Competing interests $\{28\}$}

The authors declare that they have no competing interests

\section{References}

1. Eshraghi AA, Nazarian R, Telischi FF, Rajguru SM, Truy E, Gupta C. The cochlear implant: historical aspects and future prospects. Anat Rec (Hoboken). 2012;295(11):1967-80.

2. Skarzynski H, Lorens A, Matusiak M, Porowski M, Skarzynski PH, James CJ. Cochlear implantation with the nucleus slim straight electrode in subjects with residual low-frequency hearing. Ear and hearing. 2014;35(2):e33-43.

3. Gifford RH, Dorman MF, Skarzynski H, Lorens A, Polak M, Driscoll CL, et al. Cochlear implantation with hearing preservation yields significant benefit for speech recognition in complex listening environments. Ear Hear. 2013;34(4):413-25.

4. Buechner A, Brendel M, Krueger B, Frohne-Buchner C, Nogueira W, Edler B, et al. Current steering and results from novel speech coding strategies. Otol Neurotol. 2008;29(2):203-7.

5. Gfeller KE, Olszewski C, Turner C, Gantz B, Oleson J. Music perception with cochlear implants and residual hearing. Audiol Neurootol. 2006;11 Suppl 1:12-5. 
6. Havenith S, Lammers MJW, Tange RA, Trabalzini F, della Volpe A, van der Heijden GJMG, et al. Hearing preservation surgery: cochleostomy or round window approach? A systematic review. Otol Neurotol. 2013;34(4):667-74.

7. Wanna GB, Noble JH, Carlson ML, Gifford RH, Dietrich MS, Haynes DS, et al. Impact of electrode design and surgical approach on scalar location and cochlear implant outcomes. Laryngoscope. 2014;124 Suppl 6:S1-7.

8. Wanna GB, Noble JH, Gifford RH, Dietrich MS, Sweeney AD, Zhang D, et al. Impact of intrascalar electrode location, electrode type, and angular insertion depth on residual hearing in cochlear implant patients: preliminary results. Otol Neurotol. 2015;36(8):1343-8.

9. Fan XT, Xia M, Wang Z, Zhang H, Liu CC, Wang N, et al. Comparison of electrode position between round window and cochleostomy inserting approaches among young children: a cone-beam computed tomography study. Acta Oto-Laryngol. 2018;138(9):815-21.

10. Snels C, IntHout J, Mylanus E, Huinck W, Dhooge I. Hearing preservation in cochlear implant surgery: a meta-analysis. Otol Neurotol. 2019;40(2):145-53.

11. Avci E, Nauwelaers T, Hamacher V, Kral A. Three-dimensional force profile during cochlear implantation depends on individual geometry and insertion trauma. Ear Hear. 2017;38(3):e168-e79.

12. Escudé B, James C, Deguine O, Cochard N, Eter E, Fraysse B. The size of the cochlea and predictions of insertion depth angles for cochlear implant electrodes. Audiol Neurootol. 2006;11 Suppl 1:27-33.

13. Rask-Andersen H, Erixon E, Kinnefors A, Lowenheim H, Schrott-Fischer A, Liu W. Anatomy of the human cochlea--implications for cochlear implantation. Cochlear Implants Int. 2011;12 Suppl 1:S813.

14. O'Connell BP, Hunter JB, Wanna GB. The importance of electrode location in cochlear implantation. Laryngoscope Investig Otolaryngol. 2016;1(6):169-74.

15. Holden LK, Finley CC, Firszt JB, Holden TA, Brenner C, Potts LG, et al. Factors affecting open-set word recognition in adults with cochlear implants. Ear Hear. 2013;34(3):342-60.

16. Shaul C, Dragovic AS, Stringer AK, O'Leary SJ, Briggs RJ. Scalar localisation of peri-modiolar electrodes and speech perception outcomes. J Laryngol Otol. 2018;132(11):1000-6.

17. O'Connell BP, Cakir A, Hunter JB, Francis DO, Noble JH, Labadie RF, et al. Electrode location and angular insertion depth are predictors of audiologic outcomes in cochlear implantation. Otol Neurotol. 2016;37(8):1016-23.

18. van der Jagt MA, Briaire JJ, Verbist BM, Frijns JH. Comparison of the hiFocus mid-Scala and hiFocus $1 \mathrm{j}$ electrode array: angular insertion depths and speech perception outcomes. Audiol Neurootol. 2016;21(5):316-25.

19. van der Marel KS, Briaire JJ, Verbist BM, Muurling TJ, Frijns JH. The influence of cochlear implant electrode position on performance. Audiol Neurootol. 2015;20(3):202-11.

20. Fabie JE, Keller RG, Hatch JL, Holcomb MA, Camposeo EL, Lambert PR, et al. Evaluation of outcome variability associated with lateral wall, mid-scalar, and perimodiolar electrode arrays when controlling for preoperative patient characteristics. Otol Neurotol. 2018;39(9):1122-8. 
21. Moran M, Vandali A, Briggs RJS, Dettman S, Cowan RSC, Dowell RC. Speech perception outcomes for adult cochlear implant recipients using a lateral wall or perimodiolar array. Otol Neurotol. 2019;40(5):608-16.

22. Doshi J, Johnson P, Mawman D, Green K, Bruce IA, Freeman S, et al. Straight versus modiolar hugging electrodes: does one perform better than the other? Otol Neurotol. 2015;36(2):223-7.

23. Zou J, Lahelma J, Koivisto J, Dhanasingh A, Jolly C, Aarnisalo A, et al. Imaging cochlear implantation with round window insertion in human temporal bones and cochlear morphological variation using high-resolution cone beam CT. Acta Otolaryngol. 2015;135(5):466-72.

24. Saeed SR, Selvadurai D, Beale T, Biggs N, Murray B, Gibson P, et al. The use of cone-beam computed tomography to determine cochlear implant electrode position in human temporal bones. Otol Neurotol. 2014;35(8):1338-44.

25. Mosnier I, Célérier C, Bensimon JL, de Seta D, Sterkers O, Nguyen Y, et al. Cone beam computed tomography and histological evaluations of a straight electrode array positioning in temporal bones. Acta Otolaryngol. 2017;137(3):229-34.

26. Choudhury B, Fitzpatrick DC, Buchman CA, Wei BP, Dillon MT, He S, et al. Intraoperative round window recordings to acoustic stimuli from cochlear implant patients. Otol Neurotol. 2012;33(9):1507-15.

27. Dalbert A, Sim JH, Gerig R, Pfiffner F, Roosli C, Huber A. Correlation of electrophysiological properties and hearing preservation in cochlear implant patients. Otol Neurotol. 2015;36(7):1172-80.

28. Giardina CK, Brown KD, Adunka OF, Buchman CA, Hutson KA, Pillsbury HC, et al. Intracochlear electrocochleography: response patterns during cochlear implantation and hearing preservation. Ear Hear. 2019;40(4):833-48.

29. Fontenot TE, Giardina CK, Dillon M, Rooth MA, Teagle HF, Park LR, et al. Residual cochlear function in adults and children receiving cochlear implants: correlations with speech perception outcomes. Ear Hear. 2019;40(3):577-91.

30. Skarzynski H, van de Heyning P, Agrawal S, Arauz SL, Atlas M, Baumgartner W, et al. Towards a consensus on a hearing preservation classification system. Acta Otolaryngol Suppl. 2013(564):3-13.

31. Li G. Patient radiation dose and protection from cone-beam computed tomography. Imaging Sci Dent. 2013;43(2):63-9.

32. Nardi C, Salerno S, Molteni R, Occhipinti M, Grazzini G, Norberti N, et al. Radiation dose in non-dental cone beam CT applications: a systematic review. Radiol Med. 2018;123(10):765-77.

33. Casselman JW, Gieraerts K, Volders D, Delanote J, Mermuys K, De Foer B, et al. Cone beam ct: nondental applications. JBR-BTR. 2013;96(6):333-53.

34. Drennan WR, Anderson ES, Won JH, Rubinstein JT. Validation of a clinical assessment of spectralripple resolution for cochlear implant users. Ear Hear. 2014;35(3):e92-8.

35. Manjaly JG, Nash R, Ellis W, Britz A, Lavy JA, Shaida A, et al. Hearing preservation with standard length electrodes in pediatric cochlear implantation. Otol Neurotol. 2018;39(9):1109-14. 
36. Rader T, Bohnert A, Matthias C, Koutsimpelas D, Kainz MA, Strieth S. Hearing preservation in children with electric-acoustic stimulation after cochlear implantation: outcome after electrode insertion with minimal insertion trauma. HNO. 2018;66(Suppl 2):56-62.

37. Sierra C, Calderon M, Barcena E, Tisaire A, Raboso E. Preservation of residual hearing after cochlear implant surgery with deep insertion electrode arrays. Otol Neurotol. 2019;40(4):e373-e80.

\section{Figures}

\begin{tabular}{|c|c|c|c|c|c|c|c|c|}
\hline \multirow{3}{*}{\begin{tabular}{|l|} 
CIPRES TRIAL \\
TIMEPOINT \\
\end{tabular}} & \multicolumn{8}{|c|}{ STUDY PERIOD } \\
\hline & \multirow{2}{*}{$\begin{array}{l}\text { Enrolment } \\
-2 w k \text {. }\end{array}$} & \multirow{2}{*}{$\begin{array}{l}\text { Allocation } \\
0\end{array}$} & \multicolumn{5}{|c|}{ Post-allocation } & \multirow{2}{*}{$\begin{array}{l}\text { Close-out } \\
140 .\end{array}$} \\
\hline & & & Day 0 & $\begin{array}{l}\text { wk. } \\
4-6\end{array}$ & $\begin{array}{l}\text { mo. } \\
3-4\end{array}$ & $\begin{array}{l}m_{6-7} . \\
\text {. }\end{array}$ & $\begin{array}{l}m_{13-14} \\
\text { i3 }\end{array}$ & \\
\hline \multicolumn{9}{|l|}{ ENROLMENT: } \\
\hline \multicolumn{9}{|l|}{ Eligibility screen } \\
\hline \multicolumn{9}{|l|}{ Informed consent } \\
\hline \multicolumn{9}{|l|}{ Allocation } \\
\hline \multicolumn{9}{|l|}{ Surgery } \\
\hline \multicolumn{9}{|l|}{ Cone beam $\mathrm{CT}$} \\
\hline \multicolumn{9}{|l|}{ Activation of $\mathrm{Cl}$} \\
\hline \multicolumn{9}{|l|}{ 1st audiometry/ECoch } \\
\hline \multicolumn{9}{|l|}{ 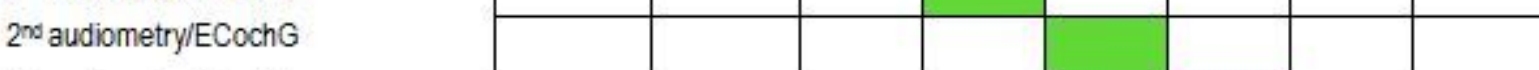 } \\
\hline \multicolumn{9}{|l|}{$3 x$ audiometry/ECoch } \\
\hline \multicolumn{9}{|l|}{$4^{\text {nh }}$ audiometry/ECoch } \\
\hline \multicolumn{9}{|l|}{ INTERVENTIONS: } \\
\hline \multicolumn{9}{|l|}{ Intervention $A,(R W / L W)$} \\
\hline \multicolumn{9}{|l|}{ Intervention $B,(R W / P M)$} \\
\hline \multicolumn{9}{|l|}{ Intervention $\mathrm{C},(\mathrm{CO} / \mathrm{LW})$} \\
\hline \multicolumn{9}{|l|}{ Intervention $\mathrm{D},(\mathrm{CO} / \mathrm{PM})$} \\
\hline \multicolumn{9}{|l|}{ ASSESSMENTS: } \\
\hline \multicolumn{9}{|l|}{ Baseline variables } \\
\hline \\
\hline & \multicolumn{8}{|c|}{ Secondary Outcomes: } \\
\hline \multicolumn{9}{|l|}{ Intraoperative $E \operatorname{Coch} G$} \\
\hline Clposition & & & & & & & & \\
\hline Post-op ECochG & & & & & & & & \\
\hline Speech perception & & & & & & & & \\
\hline
\end{tabular}

\section{Figure 1}

Schedule of enrolment, interventions, and assessments adapted from the Standard Protocol Items: Recommendations for Interventional Trials (SPIRIT). Abbreviations: Cl, cochlear implant; CO, 
Cochleostomy; CT, computed tomography; ECochG, electrocochleography; LW, lateral wall; PM, perimodiolar; PTA, pure tone audiometry; RW, round window.

\section{Supplementary Files}

This is a list of supplementary files associated with this preprint. Click to download.

- InformationletterlC.pdf 